\title{
CONTROLE DE UM AMORTECEDOR MAGNETO-REOLÓGICO EM UMA PRÓTESE DE PERNA MECÂNICA
}

\author{
Cesar H. VAlencia ${ }^{1}$, Marley M.B.R. Vellasco ${ }^{1}$, Ricardo TANSCheiT ${ }^{1}$. \\ 1. Laboratório Inteligência Computacional \\ Programa de Pós-Graduação em Engenharia Elétrica \\ Pontifícia Universidade Católica do Rio de Janeiro \\ R. Marquês de São Vicente, 225. Prédio Cardeal Leme, Sl 612.

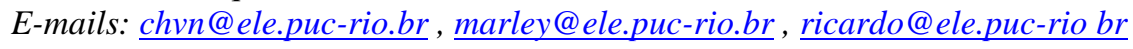

\begin{abstract}
Different models of controllers has proven to be feasible for specific tasks in equipment designed for people with physical disabilities, this paper presents and evaluates the design and simulation of a fuzzy controller for a damper MagnetoRheological embarked on a Prosthetic Leg Mechanics. As additional item was applied to characterize the behavior of the damper in a fuzzy inference system to evaluate its behavior within the proposed control system. The ultimate goal of the control is to decrease the force exerted by the knee, it is used to the full dynamic range of the AMR. The extraction of fuzzy rules for the controller used the behavior of the angle of the knee, the knee strength and percentage of gait and for extracting rules were considered MR damper characteristics of the dynamic response as the strength, the power and the speed of the piston.
\end{abstract}

Keywords_ Fuzzy Control, Magnetorheological Damper, Human Gait, Mechanical Prosthesis leg.

Resumo- Diferentes modelos de controladores tem provado ser viáveis para tarefas especificas em equipamentos desenvolvidos para pessoas com deficiências físicas, este trabalho avalia e apresenta o projeto e simulação de um Controlador Fuzzy para um amortecedor Magneto-Reológico embarcado em uma Prótese de Perna Mecânica. Como item adicional foi realizado a caracterização do comportamento do amortecedor em um sistema de inferência Fuzzy para avaliar seu comportamento dentro do sistema de controle proposto. O objetivo final do controle é a diminuição da força exercida pelo joelho, para isso é utilizado toda a faixa dinâmica do AMR. A extração de regras para o Controlador Fuzzy utilizou o comportamento do angulo do joelho, a força no joelho e a percentagem da marcha e para a extração de regras do Amortecedor MR foram considerados as características da resposta dinâmica como a força, a corrente e a velocidade de deslocamento do pistão.

Palavras-chave— Controle Fuzzy, Amortecedor Magneto-Reológico (AMR), Marcha Humana, Prótese de Perna Mecânica.

\section{Introdução}

Os avanços tecnológicos propõem novos desafios na instrumentação e controle de sistemas, o estudo de caso considerado neste trabalho faz uso da crescente necessidade de desenvolver modelos que tenham a capacidade de se adaptar e responder de forma adequada.

$\mathrm{O}$ artigo expõe uma proposta de controle utilizando a Lógica Fuzzy para uma prótese de perna mecânica utilizada por pessoas com deficiências, as próteses de baixo custo em geral não tem sistemas de amortecimento dinâmicos e aquelas que consideram este fator tem um valor muito elevado, realizando o projeto do controlador para embarcar na prótese um Amortecedor Magneto-Reológico seria possível oferecer uma alternativa com maior conforto para o usuário final.

O projeto do controlador foi realizado considerando a modelagem da marcha humana que é recorrente. Têm-se diferentes formas de subdividir a marcha, para o presente trabalho, foram divididos os estágios da marcha em quatro, segundo o modelo apresentado em Bohara (2006).

A utilização do Amortecedor MagnetoReológico foi baseada na possibilidade de aproveitar as propriedades do fluido Magneto-Reológico cuja viscosidade é controlada por um campo magnético. $\mathrm{O}$ cambio de estado se produz em um tempo pequeno o que permite um controle preciso.

No capítulo 2 é apresentada a formulação do problema; a modelagem do controlador que considera os estágios de marcha e as características do amortecedor são apresentadas no capítulo 3; os resultados obtidos para as simulações realizadas são apresentados no capítulo 4 e finalmente no capítulo 5 são expostas as conclusões do trabalho desenvolvido.

\section{Formulação do problema}

O AMR apresenta a vantagem de ser um amortecedor semiativo, ou seja, combina as propriedades dos amortecedores passivos por requer uma baixa energia para seu controle e a vantagem dos amortecedores ativos por ter uma resposta dinâmica, seu controle é uma tarefa difícil devido a sua alta não linearidade. Os algoritmos de controle tradicionais para o AMR são de alta complexidade e sua implementação requer uma carga computacional considerável, é por isso que controladores que utilizam métodos de inteligência computacional como os expostos em Atray (2003), Schurter (2000-2001) e em (Jang 1995) são desenvolvidos obtendo resultados satisfatórios. 
Os objetivos propostos neste trabalho são Gerar um Controlador Fuzzy para um Amortecedor Magneto-Reológico utilizado em próteses de pernas mecânicas e Validar o modelo Fuzzy do Amortecedor Magneto-Reológico para observar nele a força de saída para o nível de corrente resultante do controlador.

\section{Modelagem do Controlador Fuzzy}

Para ser realizada a modelagem do controlador se estabeleceu como objetivo diminuir a força no joelho naqueles pontos que é máxima e não modificar a força no joelho nos pontos onde esta é igual a 0 , para isto formam utilizados os valores de ângulo e força no joelho para os 4 modos da marcha humana segundo Bohara (2006).

A seguir são apresentadas as variáveis de entrada utilizadas, as características do amortecedor magneto-reológico e o projeto do controlador.

\subsection{Estágios da Marcha Humana}

Na Figura 1, são apresentados os 4 modos da marcha humana identificados em Bohara (2006), sendo eles modo1 "Stance Flexion/Extension", modo 2 "PreSwing", modo 3 "Swing Flexion" e modo 4 "Swing Extension".

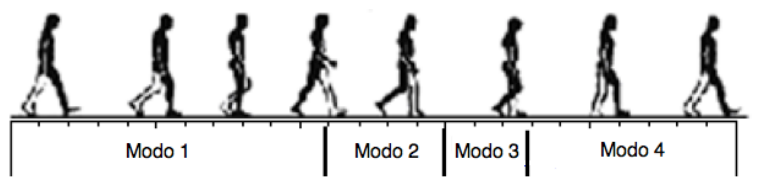

Figura 1. Modos de marcha. Adaptado de (Bohara, 2006)

A modelagem matemática apresentada em Uyar (2009) serve de referência, no comportamento da marcha humana, na equação 1 é apresentado o modelo de Uyar.

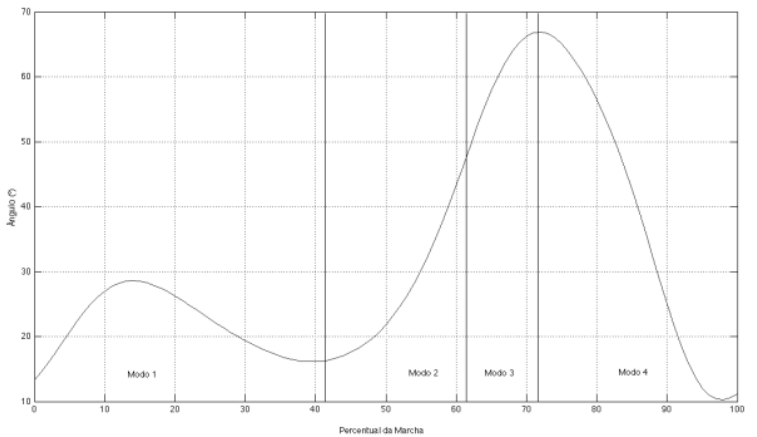

Figura 2. Ângulo do Joelho. Adaptado de (Bohara, 2006)

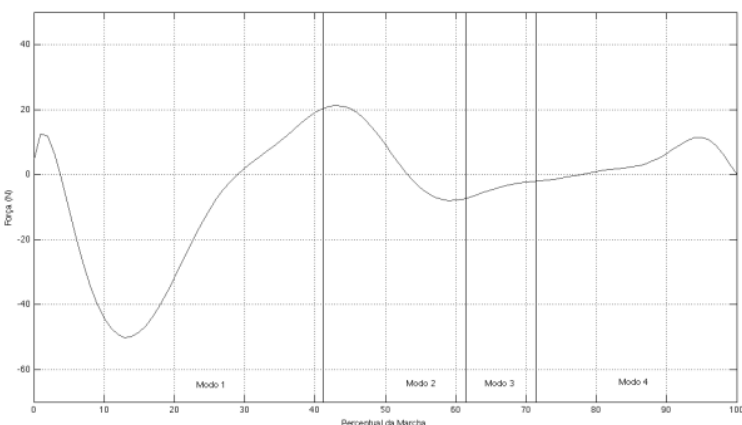

Figura 3. Força do Joelho. Adaptado de (Bohara, 2006)

As experiências realizadas para obter ditas curvas foram realizadas em sujeitos com $75 \mathrm{Kg}$ de peso em uma passada ou ciclo de marcha completa.

\subsection{Resposta do amortecedor magneto-reológico}

A utilização de um amortecedor magneto-reológico permite aproveitar a vantagem que ele gera pelo conteúdo de partículas férreas no fluido, assim, quando estas são expostas a um campo magnético são alinhadas formado cadeias que mudam a consistência do fluido, na figura 4 são apresentados os 3 estados das partículas ferrosas quando expostas a um campo magnético.

$$
\begin{aligned}
& {\left[\begin{array}{cc}
J_{0}+J_{1}+2 J_{1} \cos \theta_{2} & -J_{1} \cos \theta_{2}-J_{1} \cos \theta_{2} \\
-J_{1} \cos \theta_{2}-J_{3} & J_{3}
\end{array}\right]\left[\begin{array}{c}
\bar{\theta}_{1} \\
\theta_{2}
\end{array}\right.} \\
& {\left[\begin{array}{c}
-2 J_{1} \dot{\theta}_{1} \dot{\theta}_{2} \sin \theta_{2}+J_{1} \dot{\theta}_{2} \sin \theta_{2} \\
\dot{\theta}_{1} \dot{\theta}_{2} \sin \theta_{2}
\end{array}\right]+} \\
& {\left[\begin{array}{c}
G_{1} \sin \theta_{1}+G_{2} \sin \left(\theta_{1}-\theta_{2}\right) \\
-G_{2} \sin \left(\theta_{1}-\theta_{2}\right)
\end{array}\right]=\left[\begin{array}{l}
T_{1} \\
T_{2}
\end{array}\right]}
\end{aligned}
$$

Onde, $\mathrm{J}_{0}, \mathrm{~J}_{1}, \mathrm{~J}_{2}$ e $\mathrm{J}_{3}$ são os parâmetros da energia cinética e $\mathrm{G}_{1}$ e $\mathrm{G}_{2}$ são os parâmetros de energia potencial em uma análise da dinâmica da marcha.

Nas Figuras 2 e 3 são apresentadas as curvas de ângulo e força no joelho, sendo estas definidas nos 4 modos da marcha.

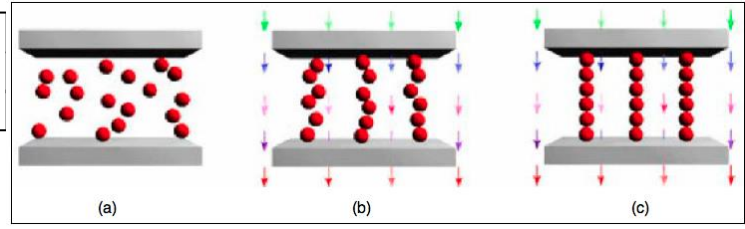

Figura 4. Ativação do fluido. (Koo, 2003)

$\mathrm{Na}$ Figura 5, é apresentada a composição do AMR, onde a haste do pistão realiza os movimentos de compressão e expansão que serão controlados na prótese mecânica. 


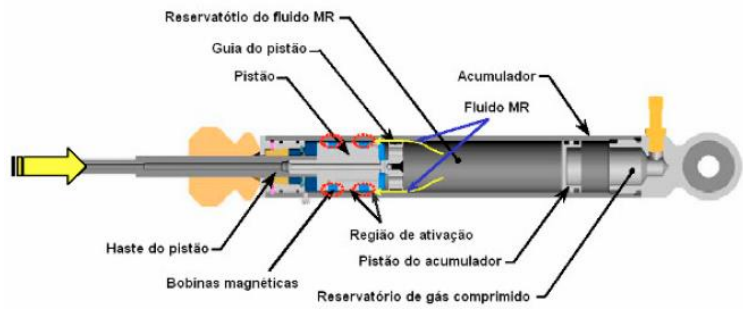

Figura 5. Amortecedor Magneto-Reológico. (Koo, 2003)

Tentando descrever as características nãolineares do AMR, foram selecionados 3 modelos matemáticos que consideram diferentes variáveis para obter a dinâmica, o primeiro deles é o Milecki $\boldsymbol{e}$ Sedziak, que é apresentado na equação 2.

$$
\begin{aligned}
& F_{d}(s)=\left(\left(k_{v} \mu\right)+D_{t l}\right) v(s) \\
& {\left[\frac{k_{h}}{\left(T_{m} s+1\right)\left(T_{e} s+1\right)} U(s)+F_{t m} e^{-\beta|v(s)|}\right]} \\
& \operatorname{sgn}[V(s)]
\end{aligned}
$$

Onde, são consideradas as características de atrito não-linear, as não-linearidades do campo magnético e os atributos do fluido magneto-reológico. No modelo de Bingham é previsto um perfil newtoniano depois do escoamento, levando em consideração o coeficiente de amortecimento viscoso e a amplitude da força de atrito de Coulomb, como é apresentado na equação 3 .

$$
F=f_{c} \operatorname{sgn}(\dot{x})+c_{o} \dot{x}+f_{o}
$$

Finalmente o modelo de Bingham modificado ou viscoelastoprástico apresentado nas equações 4 e 5 , leva em consideração o atrito, os elementos do modelo anterior mais o coeficiente de rigidez e as velocidades de deslocamento.

$$
\begin{gathered}
F=k_{1}\left(x_{2}-x_{1}\right)+c_{1} \dot{x}+f_{o} \\
F=k_{2}\left(x_{3}-x_{2}\right)+f_{o}
\end{gathered}
$$

Na Figura 6 é apresentada a dinâmica do amortecedor levando em consideração a velocidade de deslocamento de pistão, a corrente nele aplicada e a força gerada.

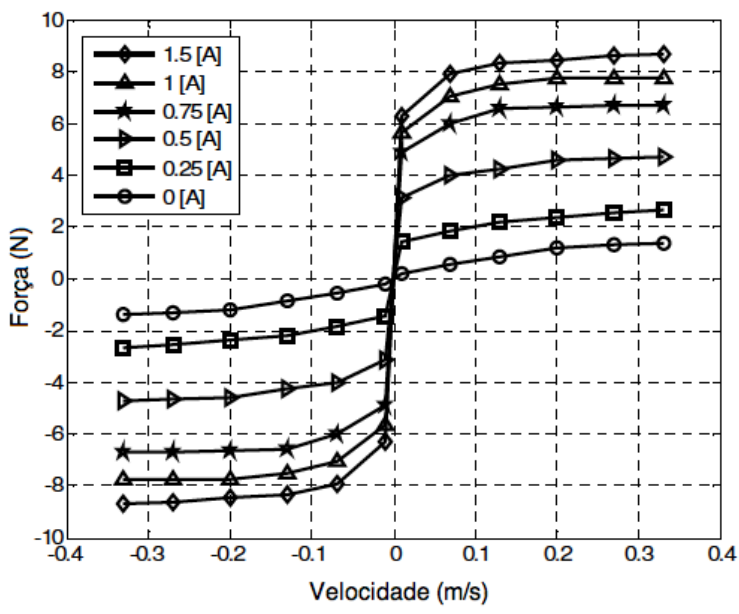

Figura 6. Dinâmica do Amortecedor MR. (Tusset, 2008)

Na figura 7, é apresentado um exemplo de um AMR embebido em uma prótese mecânica "C-Leg do Otto-Bock" para absorver os impactos no joelho.

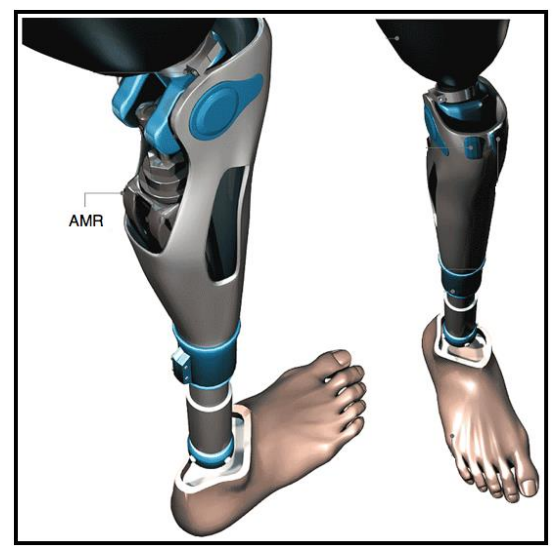

Figura 7. AMR embarcado em uma prótese mecânica.

\subsection{Projeto do controlador}

O projeto do controlador utilizando lógica Fuzzy procura exercer domínio em uma variável do amortecedor, neste caso dita variável linguística de saída será a corrente, como variáveis de entrada foram consideradas o Estagio da Marcha, o Ângulo e a Força.

Na figura 8 é apresentado o esquemático do controlador projetado. Dito controlador tem as seguintes configurações:

- Tipo de Inferência Fuzzy: Mamdani.

- Operador “or”: Mínimo.

- Operador "and": Máximo.

- Defuzzificação: Centro de Massa.

- Método de Implicação: Mínimo.

- Método de Agregação: Máximo. 


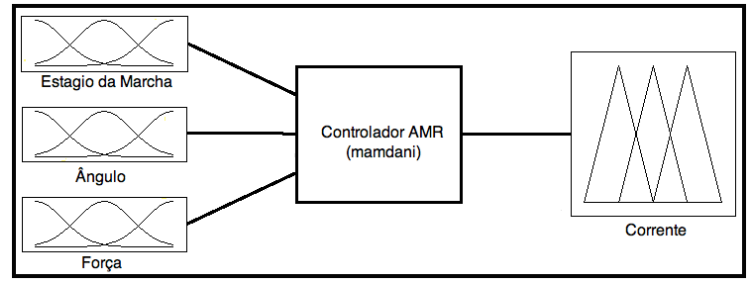

Figura 8. Diagrama de blocos do controlador

Na Figura 9 é apresentada a variável estagio de marcha, esta faz referência aos modos descritos anteriormente, isto é, 6 termos "Esti", "Est1", "Est2", "Est3", "Est4" e "Estf" onde o universo de discurso abrange uma passada que é um ciclo completo da marcha.

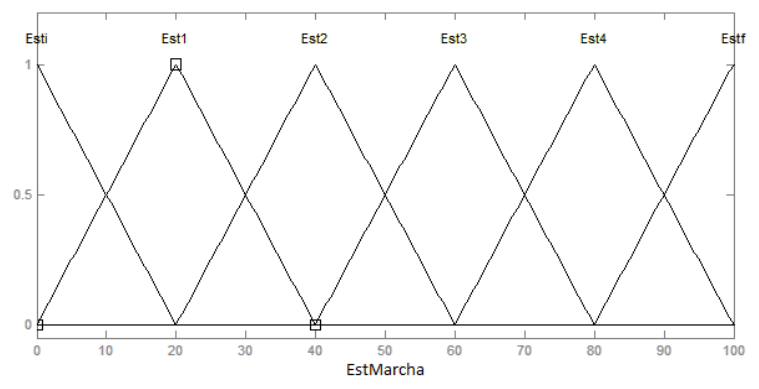

Figura 9. Conjuntos da variável de entrada "Estagio da marcha"

Na figura 10 são apresentados o termos da variável ângulo, são em total 6 "Angi" "Mbaixo", "Baixo", "Medio", "Alto" e "Angf" com um universo de discurso de 0 até 80 graus que são os valores apresentados na curva característica da marcha humana.

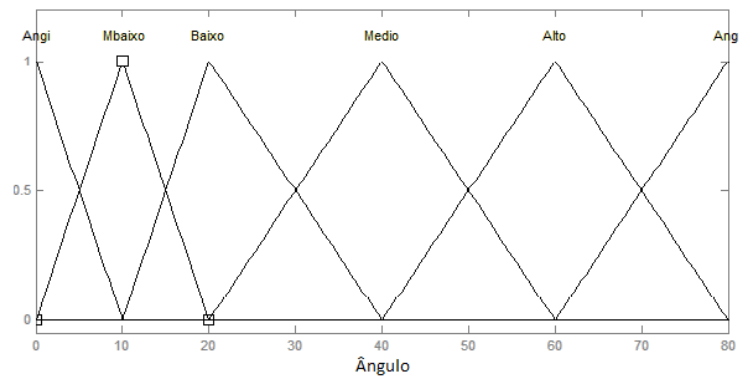

Figura 10. Conjuntos da variável de entrada "Ângulo"

Na figura 11 são apresentados os 7 termos "Fi", "Mbaixa", "Baixa", "Media", "Alta", "Malta" e "Ff", estes representam o comportamento da Força em Newtons desde $-70 \mathrm{~N}$ até $40 \mathrm{~N}$, os valores foram adotados da curva característica de força anteriormente mencionada.

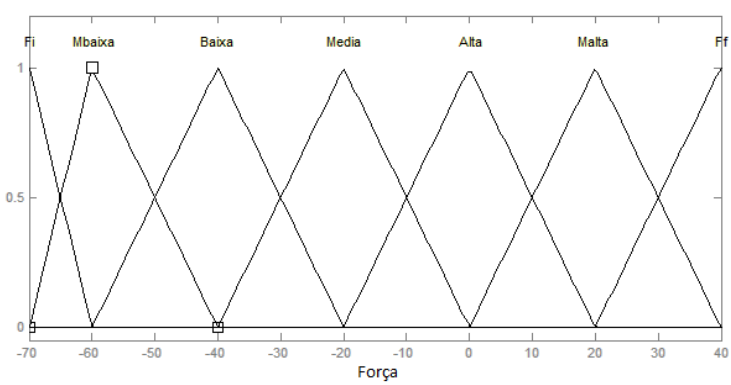

Figura 11. Conjuntos da variável de entrada "Força"

Na Figura 12 são apresentados os termos utilizados na variável de saída Corrente, em total são 13 termos que representam os valores característicos que podem ser utilizados no AMR.

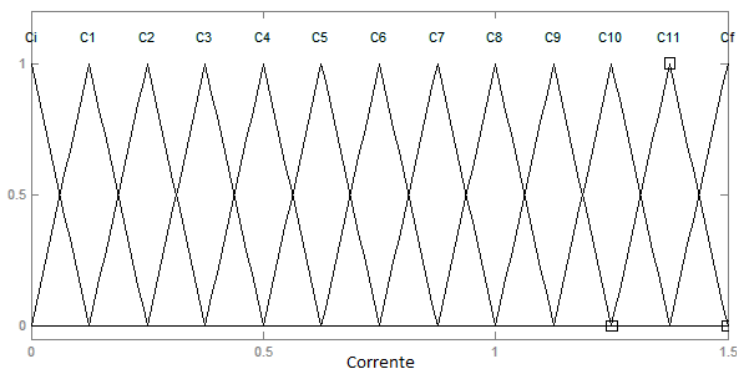

Figura 12. Conjuntos da variável de saída "Corrente"

Foram utilizadas 82 regras visando atingir o proposito inicialmente exposto, "diminuir a força no joelho naqueles pontos em que é máxima e não modificar a força no joelho nos pontos onde esta é igual a 0",

Na Figura 13 é apresentada a superfície de controle obtida, é possível observar que se tem regiões onde a corrente é 0 , isto para garantir a não interferência do AMR na marcha quando se tem valores de força iguais a 0 .

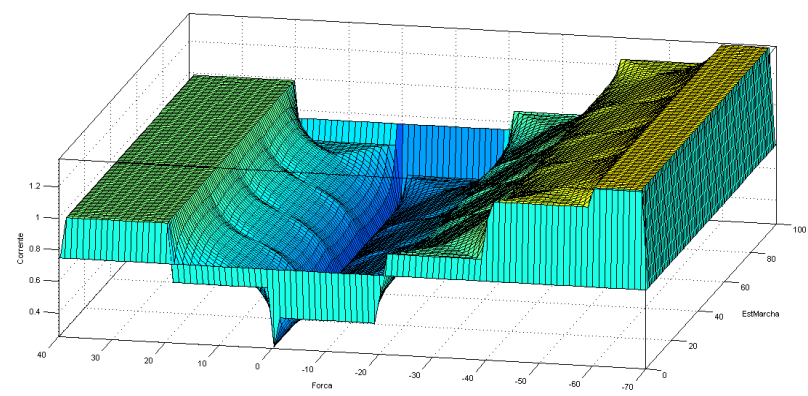

Figura 13. Corrente vs Força vs Estagio de marcha

\subsection{Modelagem Fuzzy do AMR}

Com o intuito de avaliar o desempenho de uma modelagem alternativa Fuzzy do AMR, foi proposto o sistema de inferência apresentado na figura 14 , a seguir são apresentadas as características de configuração. 
- Tipo de Inferência Fuzzy: Mamdani.

- Operador “or": Mínimo.

- Operador "and": Máximo.

- Defuzzificação: Centro de Massa.

- Método de Implicação: Mínimo.

- Método de Agregação: Máximo.

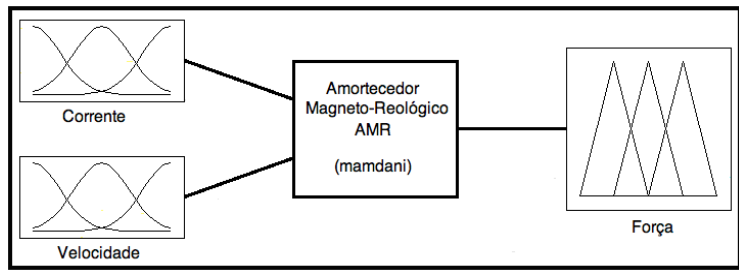

Figura 14. Diagrama de blocos da modelagem do AMR

Como variáveis linguísticas de entrada foram utilizadas a Corrente com 6 conjuntos e a Velocidade de deslocamento do pistão com 5 conjuntos e como variável de saída a Força com 7 conjuntos.

Foram geradas em total 25 regras para realizar a modelagem do AMR, na figura 15 é apresentada a superfície de controle obtida.

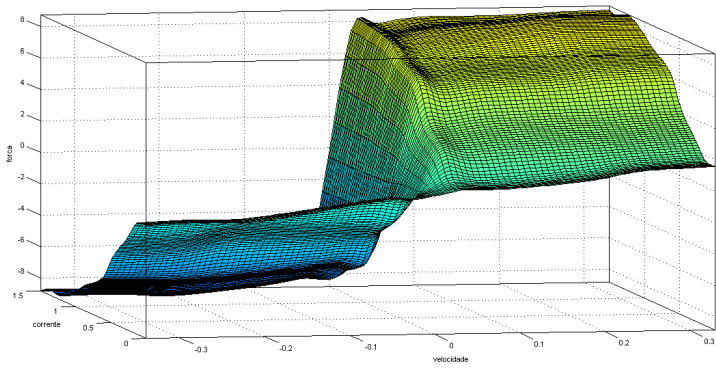

Figura 15. Força vs Velocidade vs Corrente

Os valores apresentados na superfície de controle são coerentes com a resposta dinâmica do AMR que foi apresentada na figura 6.

\section{Resultados Obtidos}

Nos testes realizados utilizando o digrama de blocos da figura 16, na modelagem do AMR foram utilizadas duas configurações, a primeira delas foi com o modelo Bingham modificado que obteve a melhor resposta dos modelos apresentados no capítulo 2 .

A segunda das configurações utilizou a modelagem Fuzzy do AMR.

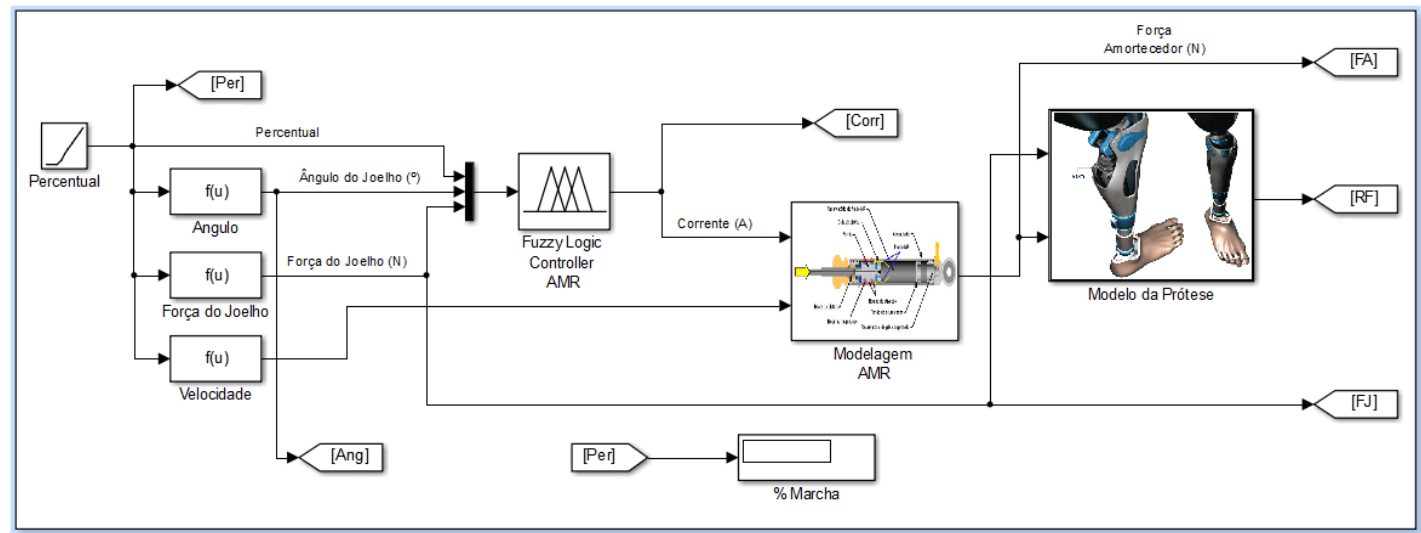

Figura 16. Diagrama do controlador proposto

Na figura 17 são apresentados os sinais fornecidos como entradas para o controlador, nela se tem o percentual de marcha, o ângulo e a força do joelho.

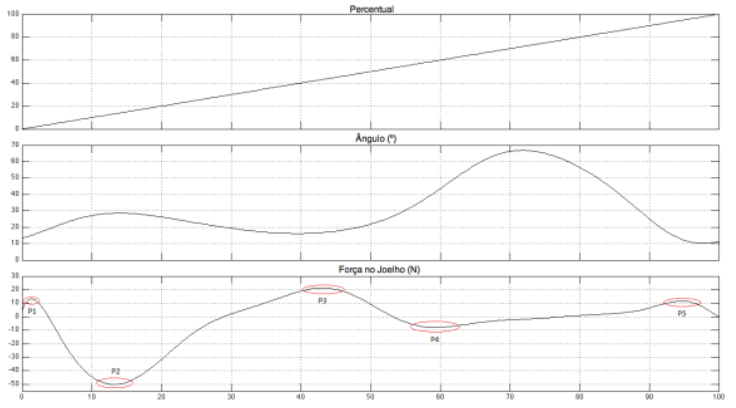

Figura 17. Diagrama de blocos da modelagem do AMR
O primeiro resultado a ser exposto é a resposta utilizando um controlador PID (PID-AMR), este controlador foi desenvolvido em trabalhos anteriores e apresentou diminuição da força nos pontos selecionados, a resposta é apresentada na figura 18.

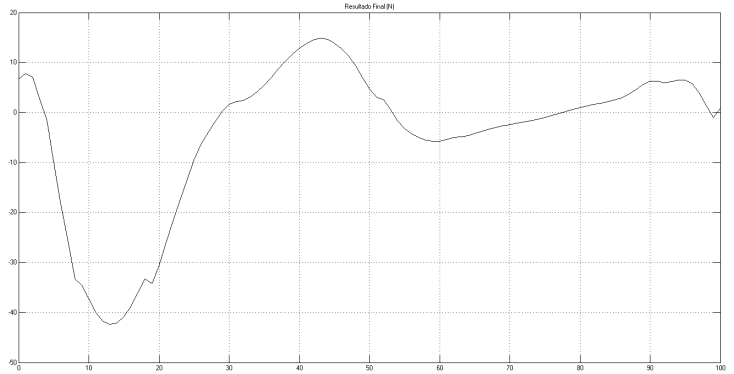

Figura 18. Resposta (PID-AMR). 
Na figura 19 é apresentado o resultado utilizando o controlador Fuzzy e o modelo Bingham modificado (C-BM-AMR), nele foi possível observar que os resultados obtidos para os pontos selecionados foram melhores comparados com os do reste anterior.

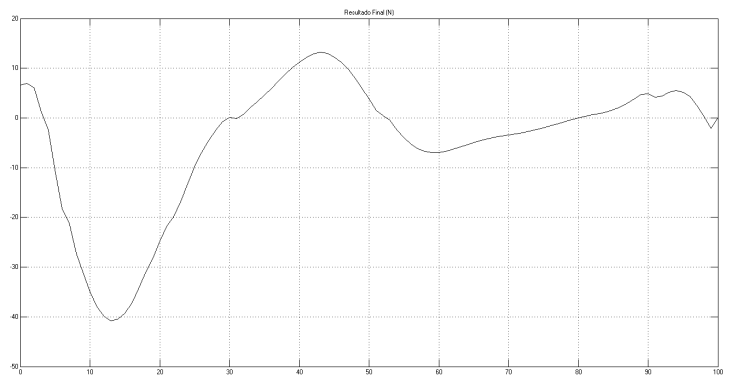

Figura 19. Resposta (C-BM-AMR)

Finalmente é apresentada na figura 20 a resposta utilizando o controlador e a modelagem $\mathrm{Fu}$ zzy do AMR, nele os resultados alcançaram os objetivos, mas, com algumas dificuldades.

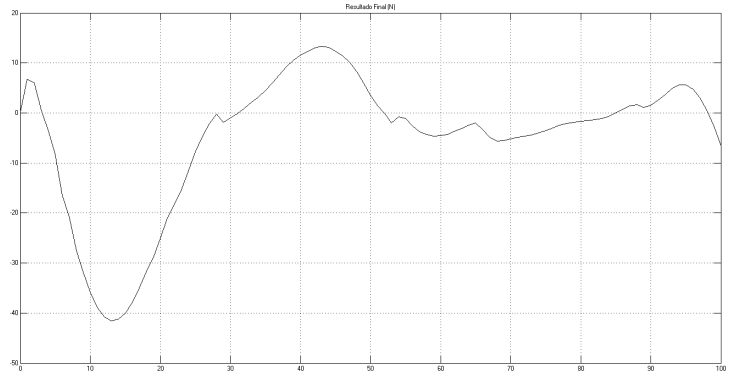

Figura 20. Resposta (C-MF-AMR)

Foram selecionados 5 pontos críticos onde o valor da força atingiu limiares que podem ser observados na figura 17.

$\mathrm{Na}$ Tabela 1 são apresentados os valores de força nos pontos críticos com o intuito de estabelecer a diferencia de desempenho dos dois modelos.

\begin{tabular}{|c|c|c|c|c|c|}
\hline Modelo & P1 & P2 & P3 & P4 & P5 \\
\hline SC-AMR & 12,4 & $-50,1$ & 21,2 & $-7,9$ & 11,5 \\
\hline PID-AMR & 7,8 & $-42,3$ & 14,8 & $-5,7$ & 6,3 \\
\hline C-BM-AMR & 6,9 & $-40,7$ & 13,2 & $-6,9$ & 5,1 \\
\hline C-MF-AMR & 6,7 & $-41,5$ & 13,3 & $-4,6$ & 5,6 \\
\hline
\end{tabular}

Os resultados obtidos para força no joelho após de utilizar a modelagem Fuzzy do AMR diminuíram nos pontos críticos selecionados de percentual de marcha $(\mathrm{P} 1 \rightarrow 2 \%, \quad \mathrm{P} 2 \rightarrow 14 \%, \quad \mathrm{P} 3 \rightarrow 44 \%$, $\mathrm{P} 4 \rightarrow 60 \%$ e $\mathrm{P} 5 \rightarrow 96 \%$ ), além, naqueles pontos iguais a zero não se teve mudanças significativas de magnitude.

\section{Conclusões}

A utilização da lógica Fuzzy no projeto de controladores estabelece um compromisso com um especialista, diversas configurações utilizadas não apresentaram os resultados esperados induzindo a reavaliar continuamente os elementos que constituem o sistema (variáveis, conjuntos e regras).

Os objetivos estabelecidos foram atingidos em ambas as configurações propostas, porém, foram observados pontos que geraram mudanças leves não desejadas, isto é, valores de força resultantes que poderiam atrapalhar a marcha.

$\mathrm{Na}$ realização do projeto do controlador foram utilizadas diferentes configurações para os conjuntos das variáveis de entrada e saída, no caso das variáveis de entrada a forma dos conjuntos afeta diretamente a região de influência das regras sendo que os conjuntos triangulares apresentaram a melhor resposta.

\section{Referências Bibliográficas}

Atray, V. S. e Roschke, P. N. «Design, fabrication, testing, and fuzzy modeling of a large magnetorheological damper for vibration control in a railcar», IEEE/ASME Joint Railroad Conference, 2003., Chicago, IL, USA, págs. 223229.

Bohara, A. «Finite State Impedance-Based Control of a Powered Transfemoral Prosthesis» Tese de Mestrado, Vanderbilt University, EUA, 2006.

Jang, J.-S. R. e Chuen-Tsai Sun, «Neuro-fuzzy modeling and control», Proceedings of the IEEE, vol. 83, págs. 378-406, Mar. 1995.

Koo, J-H. «Using Magneto-Rheological Dampers in Semiactive Tuned Vibration Absorbers to Control Structural Vibrations» Tese de Doutorado, Virginia State University, EUA, 2003.

Schurter, K. C. e Roschke, P. N. «Fuzzy modeling of a magnetorheological damper using ANFIS», in Ninth IEEE International Conference on Fuzzy Systems. FUZZ- IEEE 2000, San Antonio, TX, USA, págs. 122-127.

Schurter, K. C. e Roschke, P. N. «Neuro-fuzzy control of structures using magnetorheological dampers», in Proceedings of the 2001 American Control Conference. Arlington, VA, USA, págs. 1097-1102.

Tusset, Â. M. «Controle Ótimo Aplicado em Modelo de Suspensão Veicular Não-Linear Controlada Através de Amortecedor MagnetoReológico» Tese de Doutorado, Universidade Federal do Rio Grande do Sul, Brasil, 2008.

Uyar, E; Baser, O; Bace, R e Özçivici, E. «Investigation of bipedal human gait dynamics and knee motion control» Izmir, Turkey: Dokuz Eylül University - Faculty of Engineering Department of Mechanical Engineering. Retrieved August 2009. 